Background Patients with Type 1 diabetes should have access to $24 / 7$ specialist advice on diabetes related issues by trained health care professionals and consistent with nationally agreed guidance

Objectives

- To review the out of hours diabetes advice line for Children and Young People with diabetes over a 9 month period

- To analyse feedback from families regarding the quality of service

- To understand paediatric trainees' perspective, confidence and perception of training offered in this area.

Methods

- Recorded out of hours phone advice for Children and Young people with diabetes was reviewed over a 9 month period and results summarised.

- A survey of all paediatric trainees' level of confidence and perception of specific training offered with suggestions for future design of education package was undertaken

- A phone survey of 23 families was undertaken to seek their feedback and suggestions for improvement.

- A resource pack created within our health trust with frequently asked questions and scenarios was reviewed and further refined based on feedback

Results A total of 119 calls were recorded over a 9 month period. Average 13-calls/month. Week days $=68$ calls $(27$ between 1700-2100 and 41 after 2100). Weekends and bank holidays $=49.2$ calls. Common issues - new diagnosis checking doses, help with correction, hypos, high sugars with or without ketones, vomiting, wrong insulin given, pump failure, technical issues. Advice offered by- F2, GPST, SD, ST1-8, Consultant

35 trainee surveys were returned with $80 \%$ from level1\& 2 trainees. $74 \%$ rated their personal confidence at giving advice as 3 or below (scale of 1-5). Trainees found 'pump troubleshooting' and 'ketone management' most challenging. $62 \%$ said they received no specific teaching on managing out of hours diabetes related phone queries and would welcome creation of a resource pack with frequently asked questions and scenarios. Additionally they asked to include further scenarios such as correcting hyperglycaemia in the middle of the night, threshold for admission and specific guidance on this.

13 families said they have used the service at least 5 times or more over the last 12 months. All families were satisfied by the quality of advice and felt reassured. The Health Care Professionals were perceived as confident and time efficient. $50 \%$ of the families were calling after having recently been diagnosed with type 1 diabetes and seeking reassurance on practice or checking if dose was appropriate.

The Trust 'out of hours diabetes advice line' standard operating procedure and scenario document was disseminated by email to all Children and Young Persons diabetes teams across the region through the Regional Diabetes Managed Clinical Network for comments. Responses were received from most trusts with helpful suggestions to create a consistent training package.

Conclusions Out of hours diabetes advice line was used at least 3 to 4 times per week by families with proportionately higher use over the weekend, most commonly for a new diagnosis in type 1 diabetes. Families offered positive feedback on the quality of telephone advice. Trainees would welcome training in this area. There is an appetite for a regional training package.
British Paediatric Neurology Association

\section{KNOWLEDGE AND ATTITUDE OF THE HEALTHCARE STAFF TOWARDS EPILEPSY: EXPERIENCE FROM ONE CENTER IN THE UNITED ARAB EMIRATES}

Vivek Mundada. Aster DM Healthcare, Dubai

\subsection{6/archdischild-2021-rcpch.4}

Background Children with epilepsy along with their families often suffer from stigma and discrimination. Such stigma is mainly because of a lack of awareness and knowledge about this condition. Unfortunately, this negative attitude exists in the individuals with whom the affected person can come into contact on daily basis, such as school teachers or relatives. The impact of such stigma can be even more harmful than the disease itself, affecting the quality of life of the patients.

Objectives This study was conducted to assess the knowledge, awareness, and attitude towards epilepsy of the healthcare staff from one of the specialized hospitals in Dubai, United Arab Emirates

Methods An online survey was created using Google documents which consisted of seven sections. The first section included information about the responder's work area and designation. Sections 2 and 3 had questions assessing the basic knowledge and understanding about epileptic seizures and epilepsy; whereas sections 4 and 5 were targeted to understand the behaviour and attitude of the responder towards the person with epilepsy. The questionnaire was emailed to all the pediatric doctors and the nursing as well as administrative staff who were working in the emergency, outpatient, and inpatient settings. Out of the total 120 requests, 88 responses were collected $(73 \%)$.

Results Except for three responders, all others had mentioned that they had either heard or read about epilepsy through more than one source. For the question- 'What causes epilepsy in children?', there were options like 'blood disorders' and 'stress or mental illness' in addition to the known etiological factors as per the 2017 ILAE classification of epilepsy. $14 \%$ of the responders thought that epilepsy is caused by some blood disorders; whereas $48 \%$ thought that stress or any mental disorder cause it.

Almost $94 \%$ of the responders knew that an epileptic seizure happens due to 'abnormal electrical activity in the brain'. However, only two-third knew what an 'aura' is. Nearly half of the responders (49\%) were aware that some epilepsies can be dealt with or treated with surgical approaches. There was less awareness or acknowledgment of the other forms of seizures like autonomic or absence seizures.

A quarter of the responders thought that they could feel anxious while dealing any child with active seizures during their shift which included even the general practitioners (4\%) and staff nurses (14\%). Only 24\% did not mind their children getting married to a person with epilepsy. $24 \%$ believed that anyone with epilepsy should not be employed with a job like nursing in any hospital and $80 \%$ believed that an affected person should never consume alcohol.

The majority of the responders felt that there is still some need of acquiring more education in the field of epilepsy.

Conclusions Our survey which was conducted in a single-center highlights the knowledge gap and issues with the attitude and behaviour among healthcare professionals towards the 
individuals affected with epilepsy. We feel that there is an unmet need for ongoing education in this field which should be catered to the recipient's needs.

\section{AUDIT OF FIRST PAEDIATRIC ASSESSMENT OF CHILDREN REFERRED WITH SUSPECTED SEIZURES BEFORE AND DURING THE SARS-COV-2 PANDEMIC}

${ }^{1}$ Maymunah Khries, ${ }^{2}$ Helen Estyn-Jones. ' Great North Children's Hospital; ${ }^{2}$ Department of Paediatrics, Queen Elizabeth Hospital, Gateshead

10.1136/archdischild-2021-rcpch.5

Background Diagnosing epilepsy can be complex with misdiagnosis rates of $5-30 \%$. NICE guideline recommends all children and young people presenting with a suspected epileptic seizure to be seen by a specialist in the diagnosis and management of the epilepsies within 2 weeks of presentation. Timely specialist assessment aims to

- improve the detailed history upon which accurate diagnosis depends.

- facilitate appropriate subsequent management.

- support anxious parents.

Has Covid-19 pandemic added an extra challenge for specialist healthcare providers trying to achieve best practice?

Objectives

- To audit our specialist service for children and young people with suspected seizure(s) against NICE recommendation.

- To compare numbers of referrals and timing of first assessment during the pandemic with the same time period the year before.

- To look at the diagnostic outcomes following specialist assessment for first suspected seizure.

Methods

- Identification of 2 cohorts of children presenting to specialist outpatient service for suspected seizure between March-May 2019 and March-May 2020.

- Retrospective case notes analysis following first Paediatric assessment.

Results

- Number of referrals declined by more than $20 \%$ during the pandemic, especially from general practitioners.

- In 2019 cohort (pre-pandemic), 55\% of the cases were seen within 14 days of referral compared to $42 \%$ in 2020 cohort (during pandemic).

- More than half of the referrals were diagnosed as nonepileptic events after specialist review. However, there were less non-epileptic events diagnosed in 2020 compared to the previous year.

\section{Conclusions}

- The pandemic is likely to have reduced the number of referrals, possibly due to some families following the 'stay at home' message and not presenting at all, and GPs deciding against referral of vague or likely non-epileptic episodes.

- Delayed referral to first specialist assessment might have resulted from assumptions about service cancellations.

- Pandemic restrictions affect staffing and availability of spacious rooms for distanced consultations.

Discussion This audit examined the impact of first wave of the pandemic since when changes to our Paediatric service in general and the first seizure pathway may be helping meet the best practice challenge:

- Incorporating virtual platform in morning handover increases wider participation and opportunities to raise awareness of first seizure clinic.

- Remote access to trust systems means that referrals could be triaged quickly.

- Appointments are now mainly by 'attend anywhere' video or telephone, since event diagnosis is largely dependent on detailed history.

Further improvements are being considered:

- Work with IT to further develop electronic discharge documentation e.g. Drop-down menu of common presentations including 'first suspected seizure' with subsequent prompts such as 'eye-witness history', 'ECG' and 'first suspected seizure clinic referral'.

- Create email for epilepsy team to act as 'single point of access' to share triage, support communication and crosscover during leave.

- Continue to make investment in epilepsy service, including a Paediatric epilepsy nurse to support worried families, nonexpert colleagues and the service.

This poster could provide useful information to colleagues in primary and secondary care about our service and the clinical feedback may support training to improve confident diagnosis and management of common non-epileptic events such as syncope.

We aim to repeat this audit over the same time period in 2021.

\section{British Paediatric Allergy Immunity and Infection Group}

\section{A SERVICE EVALUATION OF PAEDIATRIC OUTPATIENT PARENTERAL ANTIBIOTIC THERAPY FOR THE MANAGEMENT OF DEEP BRAIN STIMULATION DEVICE INFECTIONS}

${ }^{1}$ Temitope Fisayo, ${ }^{2}$ Jennifer Handforth, ${ }^{3}$ Matthew Chico. 'London School of Hygiene and Tropical Medicine, King's College London; ${ }^{2}$ Evelina London Children's Hospital; ${ }^{3}$ London School of Hygiene and Tropical Medicine

\subsection{6/archdischild-2021-rcpch.6}

Background Dystonia is a movement disorder. Deep Brain Stimulation (DBS) is a surgical treatment for childhood dystonia wherein a DBS device is implanted in the brain. DBS devices may become infected. Paediatric Outpatient Parenteral Antibiotic Therapy (pOPAT) has been used to manage complex infections at home. This study evaluates the pOPAT service for the management of DBS device infections in a single centre. Objectives The goal of this project was to answer the following questions:

- Which pathogens infect the DBS devices in children who are subsequently managed by the pOPAT service?

- How are infections in children with DBS devices being managed under the pOPAT service?

- Does the pOPAT management prevent removal or revision of DBS devices following DBS device infection in children? 